\title{
Electrical Brain Stimulation Improves Cognitive Performance by Modulating Functional Connectivity and Task-Specific Activation
}

\author{
Marcus Meinzer, ${ }^{1 *}$ Daria Antonenko, ${ }^{1 *}$ Robert Lindenberg, ${ }^{1 *}$ Stefan Hetzer, ${ }^{2}$ Lena Ulm, ${ }^{1}$ Keren Avirame, ${ }^{1}$ \\ Tobias Flaisch, ${ }^{3}$ and Agnes Flöel ${ }^{1}$ \\ ${ }^{1}$ Department of Neurology, Center for Stroke Research Berlin, and Cluster of Excellence NeuroCure, Charite Universitätsmedizin, Berlin 10117, Germany, \\ ${ }^{2}$ Bernstein Center for Computational Neuroscience and Berlin Center for Advanced Neuroimaging, Berlin 10117, Germany, and ${ }^{3}$ Department of Psychology, \\ University of Konstanz, Konstanz 78464, Germany
}

Excitatory anodal transcranial direct current stimulation (atDCS) can improve human cognitive functions, but neural underpinnings of its mode of action remain elusive. In a cross-over placebo ("sham") controlled study we used functional magnetic resonance imaging (fMRI) to investigate neurofunctional correlates of improved language functions induced by atDCS over a core language area, the left inferior frontal gyrus (IFG). Intrascanner transcranial direct current stimulation-induced changes in overt semantic word generation assessed behavioral modulation; task-related and task-independent (resting-state) fMRI characterized language network changes. Improved word-retrieval during atDCS was paralleled by selectively reduced task-related activation in the left ventral IFG, an area specifically implicated in semantic retrieval processes. Under atDCS, resting-state fMRI revealed increased connectivity of the left IFG and additional major hubs overlapping with the language network. In conclusion, atDCS modulates endogenous low-frequency oscillations in a distributed set of functionally connected brain areas, possibly inducing more efficient processing in critical task-relevant areas and improved behavioral performance.

\section{Introduction}

Noninvasive transcranial direct current stimulation (tDCS) modulates brain activity by applying weak electrical currents to the scalp. Depending on the current polarity, excitability of underlying brain areas is increased (anodal tDCS, atDCS) or decreased (cathodal tDCS) by modulating resting membrane potentials (Stagg and Nitsche, 2011). The most consistent beneficial effects on motor and cognitive performance in healthy subjects and patients with neurological injury have been reported for excitatory atDCS (Flöel et al., 2008, 2011; Reis et al., 2009). Behavioral gains are independent of unspecific (e.g., attentional) effects and participants can be effectively blinded to the stimulation by comparison with placebo ("sham") stimulation (Gandiga et al., 2006). Understanding its mode of action will have major

Received Sept. 20, 2011; revised Nov. 16, 2011; accepted Dec. 12, 2011.

Author contributions: M.M. and A.F. designed research;M.M., D.A., R.L., S.H., L.U., and K.A. performed research;

T.F. contributed unpublished reagents/analytic tools; M.M., D.A., R.L., and S.H. analyzed data; M.M., R.L., T.F., and A.F. wrote the paper.

This work was supported by grants from the Deutsche Forschungsgemeinschaft (Grant FI-379-8/1 to A.F.; and by DFG-Exc-257), the Bundesministerium für Bildung und Forschung (Grant FKZ0315673A to A.F.; and Grant $01 E 00801$ to A.F. and M.M.), and the Else-Kröner Fresenius Stiftung (2009-141 to A.F.). We thank G. Lohmann for support with the LIPSIA software package.

*M.M., D.A., and R.L. contributed equally.

The authors declare no competing financial interests.

Correspondence should be addressed to either Dr. Marcus Meinzer or Prof. Agnes Flöel, Charite Universitätsmedizin Berlin, Department of Neurology, Chariteplatz 1, 10117 Berlin, Germany. E-mail: marcus.meinzer@charite.de or agnes.floeel@charite.de.

DOI:10.1523/JNEUROSCI.4812-11.2012

Copyright $\odot 2012$ the authors $\quad 0270-6474 / 12 / 321859-08 \$ 15.00 / 0$ implications for cognitive neuroscience research, and possibly enable researchers to target specific cerebral networks in patients with neurological diseases.

A number of studies reported beneficial effects of atDCS on higher cognitive functions (Antal et al., 2004; Cattaneo et al., 2011; Flöel et al., 2012), but only one study investigated the neural underpinnings of these effects (Holland et al., 2011). In this study, atDCS-induced picture naming improvement was associated with reduced activity in left inferior frontal gyrus (IFG). In the motor domain, $\mathrm{tDCS}$-induced changes of local brain activity and structurally connected remote brain areas have been described (Antal et al., 2011; Polanía et al., 2011; Zheng et al., 2011). However, a comprehensive assessment of tDCS-induced changes in task-related local activity and whole-brain functional connectivity has not been conducted for either motor or cognitive functions.

Based on previous behavioral studies demonstrating beneficial effects of atDCS on language processing (Iyer et al., 2005; Flöel et al., 2008; Cattaneo et al., 2011), we used functional magnetic resonance imaging (fMRI) during overt semantic word generation and simultaneous intrascanner atDCS to investigate effects on performance and task-related activity. Stimulation targeted the left IFG, an area critical for successful word-retrieval (Cattaneo et al., 2011). Specificity of task-related activity changes was assessed by analyzing subportions of the left and right IFG. In particular, the left ventral IFG (vIFG) has specifically been implicated with semantic retrieval processes (Thompson-Schill et al., 1997). We thus expected behavioral improvement to be associ- 

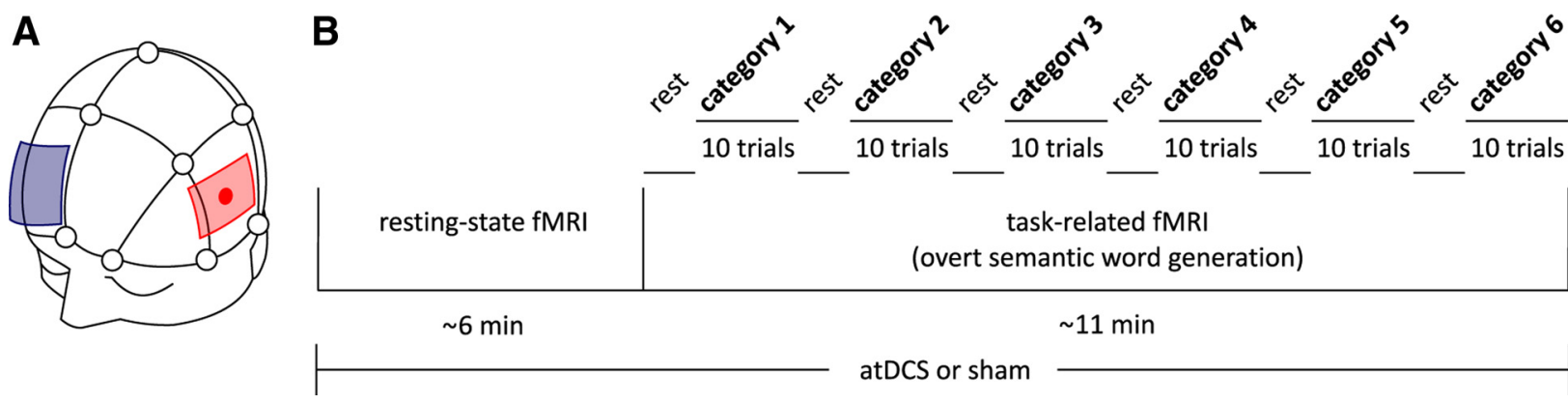

task-related fMRI

(overt semantic word generation)

$\sim 11 \mathrm{~min}$

atDCS or sham

Figure 1. Details of the study design. $\boldsymbol{A}$ illustrates the stimulation site (left IFG; red) and the position of the reference electrode (blue). Stimulation site was determined according to $10-20$ EEG-system. $\boldsymbol{B}$ shows details of an fMRI session.

ated with selectively decreased activity in the left vIFG, indicative of more efficient neural processing (Antal et al., 2011; Holland et al., 2011). In addition, task-independent resting-state fMRI investigated whole-brain functional connectivity. A graph-based data analysis approach (Eigenvector Centrality Mapping, ECM) was chosen that defines connectivity of central cortical nodes using spectral coherence patterns across the entire brain without requiring a priori assumptions (Lohmann et al., 2010a; Taubert et al., 2011). We were specifically interested in low-frequency fluctuations reflecting functionally meaningful endogenous oscillations of anatomically connected brain areas (Achard et al., 2006). Enhanced motor network connectivity has been described after atDCS over the primary motor cortex (Polanía et al., 2011). Thus, we hypothesized that atDCS would increase IFG connectivity and connectivity of structurally and functionally connected language-related areas, possibly providing a neural basis for local tDCS effects during task performance.

\section{Materials and Methods}

Twenty healthy right-handed native German speakers were recruited (10 males, 10 females; mean age \pm SD, $26.7 \pm 3.8$ years; range $19-34)$. None of the subjects reported use of psychoactive medication or recreational drugs. In a cross-over within-subjects design, participants were assessed during two identical fMRI sessions (either with concomitant atDCS or sham tDCS), separated by at least 1 week to prevent carry-over effects. During the fMRI sessions subjects participated in a resting-state sequence and a paced semantic word generation task (Meinzer et al., 2009). During word generation, participants were presented with six semantic categories and their task was to overtly generate 10 different exemplars for each category (i.e., a maximum of 60 correct responses). Two different matched sets of categories were used during the two fMRI sessions following a pilot study in a different group of subjects that assured comparability of the two sets (see below for details; Fig. 1 shows details of the design). The study was approved by the local ethics committee and conducted in accordance with the Helsinki Declaration. Written informed consent was obtained from all participants before study inclusion and they were compensated with $€ 30$.

$t D C S$. Order of stimulation was counterbalanced across subjects. Direct current was provided through a battery-driven MRI-compatible stimulator (DC-Stimulator Plus, neuroConn $\mathrm{GmbH}$ ) positioned outside the scanner room. To avoid temperature rise and to reduce induction voltages due to high radio frequency pulses, $5-\mathrm{k} \Omega$ resistors were included in each electrode cable and two filter boxes (absorbing radio frequency noise) were placed between stimulator and electrodes inside and outside the scanner. This setting has been described previously and formal testing showed no electrode artifacts and only minor overall reductions of signal-to-noise ratio. Most importantly, no differences were found between areas closer or more distant to the electrode and when comparing active versus sham stimulations (Antal et al., 2011). The stimulating electrode was inserted in a $5 \times 7 \mathrm{~cm}^{2}$ saline-soaked synthetic sponge, and centered over left Brodmann areas (BA) 44/45, as previous studies found significantly improved semantic fluency with this electrode montage (Iyer et al., 2005; Cattaneo et al., 2011). Electrode positions were individually defined according to the 10-20 EEG system. We determined (a) the intersection of T3-F3 and F7-C3 and (b) the midpoint between F7-F3. The electrode was positioned at the center of a line connecting points (a) and (b). Correct electrode positions were verified on the T1-weighted images in every subject. The reference electrode $\left(10 \times 10 \mathrm{~cm}^{2}\right)$ was positioned over the contralateral supraorbital region. The increased size of the frontopolar reference electrode renders stimulation functionally inefficient without compromising tDCS effects under the active electrode (Stagg and Nitsche, 2011). tDCS was delivered with a constant current of $1 \mathrm{~mA}$ during resting-state and task-related fMRI and continued until the end of the semantic task. For both stimulation conditions (atDCS, sham) the current was initially increased in a ramp-like fashion over $10 \mathrm{~s}$, eliciting a tingling sensation on the scalp that fades over seconds. During sham stimulation the current was turned off after $30 \mathrm{~s}$.

Stimulus selection for the semantic task. We compared the effects of atDCS on semantic word generation using 12 preselected semantic categories that were divided into two matched sets based on published norms on category sizes [Set1: trees, insects, sports equipment, body parts, beverages, occupations; Set2: flowers, fish, kitchen appliances, clothing, food, hobbies; Set1/Set2: total number of exemplars produced in norm group, 1586.0/1587.8; category size, 11.6/11.7; fluency, 0.64/0.60 (Mannhaupt, 1983)]. Additionally, a pilot study was conducted in a different group of 20 young healthy participants (10 females/males; mean $\pm \mathrm{SD}$, age $25.6 \pm 3.7$ years; range $21-34$ ) who performed a standard semantic verbal fluency task ( 1 min duration) using all 12 categories in randomized order. The number of exemplars produced by this group was also comparable between the two sets (mean \pm SD number of correct exemplars produced; Set1, $20.4 \pm 3.3$; Set2, $20.5 \pm 2.5 ; t_{(19)}=0.18, p=$ $0.85)$. During scanning, the same categories were used for all participants with order of appearance randomized within sets. The two sets were counterbalanced across the group.

MRI parameters. Scanning was conducted using a 3-Tesla Siemens Trio MR-System at the Berlin Center for Advanced Neuroimaging. For task-related BOLD fMRI a T2*-sensitive echo-planar imaging sequence at $3 \times 3 \times 3 \mathrm{~mm}^{3}$ resolution was used $(\mathrm{TR}=6000, \mathrm{TA}=2000, \mathrm{TE}=30$, flip angle: $90^{\circ}, 32$ transverse slices; gap, $0.75 \mathrm{~mm}$; slice thickness, $3 \mathrm{~mm}$; interleaved acquisition, FOV, $192 \times 192$; acquisition matrix, $64 \times 64$ ). A total of 104 functional whole-brain images were acquired during each session. Continuous resting-state BOLD-fMRI was acquired with the following parameters: resolution, $3 \times 3 \times 4 \mathrm{~mm}^{3}$; TR/TA $=2300$; $\mathrm{TE}=$ 30; flip angle, $90^{\circ}$; 34 transverse slices, no gap; interleaved acquisition, FOV, $192 \times 192$; acquisition matrix, $64 \times 64,150$ functional whole-brain volumes. Standard T1-weighted sequences with $1 \mathrm{~mm}^{3}$ isotropic voxels were also acquired to facilitate normalization.

Semantic fluency task. The task has been described previously in detail (Meinzer et al., 2009, 2012). In short, each category was visually presented in blocks of 10 consecutive trials. Participants were instructed to produce one different exemplar during each trial. Each category was displayed for $3.8 \mathrm{~s}$, during which the participants responded overtly with one exemplar of the given category. Afterward, the stimulus disappeared, 


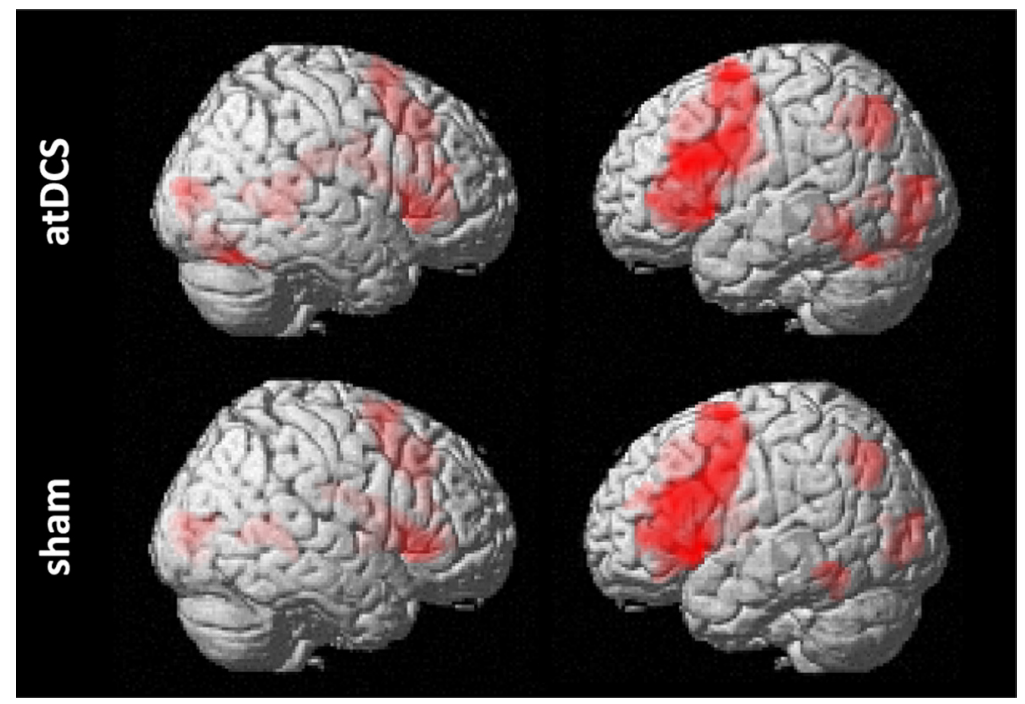

Figure 2. Illustrates task-related fMRI results. Surface renderings of bilateral activity patterns associated with correct semantic word generation trials compared with the baseline (saying "rest") for atDCS and sham (left columns = right hemisphere; right columns $=$ left hemisphere). Images are thresholded at $p=0.0005$, corrected for multiple comparisons using the false discovery rate.

was replaced by a black screen $(2.2 \mathrm{~s})$ and a single whole-brain functional MR volume was acquired $0.2 \mathrm{~s}$ after stimulus offset. Subjects were instructed to say the word 'next' if they could not come up with a correct exemplar. Task blocks alternated with a baseline condition (saying the word "rest"; five consecutive trials). Overt verbal responses were assessed in the scanner during an off-phase and the hemodynamic response was acquired after a short time delay (temporal sparse sampling) to avoid speech-related artifacts. Total duration of the experiment was $10.4 \mathrm{~min}$. Before scanning, a training session outside of the scanner using a different set of categories was performed to familiarize the participants with the task. Verbal responses were transmitted from a microphone in the scanner to a speaker, recorded and transcribed. Responses were subsequently scored for correctness by three independent raters blind to the stimulation condition. Incorrect responses (exemplars that do not belong to a given category), omissions and repetitions of an exemplar (same exemplar, synonyms) were scored as errors. In case of disagreement a consensus was reached by the three raters. Response times were determined using the recorded speech samples using Audacity sound editing software.

Task-related fMRI data analysis. Statistical parametric mapping was used for task-related fMRI data analysis (SPM5; Wellcome Department of Imaging Neuroscience, London, UK). Preprocessing comprised image realignment to compensate for head movements and coregistration of functional images with the individual subjects' anatomical image. Unified segmentation was applied to the T1-images and the resulting normalization parameters were applied to the functional images to improve registration to MNI standard space. The resulting images were spatially smoothed with a Gaussian kernel of $8 \times 8 \times 8 \mathrm{~mm}$ (FWHM). The design matrix for the statistical analysis comprised the two covariates-ofinterest (semantic word generation trials; baseline trials) as well as covariates-of-no-interest (movement parameters). Before estimation of the model a high-pass filter (cutoff period $128 \mathrm{~s}$ ) was applied to the data. Data were modeled using a finite impulse response (Gaab et al., 2007).

The effects of the conditions were determined in a single statistical model at the individual subject level to account for session-specific effects. Critically, only correct trials were included and the number of correct trials was equalized for both fMRI sessions on an individual basis to assure that potential differences between the stimulation conditions were not confounded by a different number of correct responses in the two sessions. To achieve this, correct responses from the session with the smaller number of correct responses were included in the analysis. Volumes that were at the same position in the time series in the session with the larger number of correct trials were removed from the analysis of a given subject. Incorrect and excluded trials of both scanning sessions were included as additional regressors in the statistical model. The number of trials in the respective analyses of individual subjects ranged from 44 to 54 trials (please note that previous studies using the same design only used 40 trials (Meinzer et al., 2009,2012 ), thus, it was ensured that a sufficient number of trials could be analyzed for all subjects). Planned contrasts of interest were estimated for each subject and session (semantic word generation vs baseline trials). Based on our primary hypothesis we subsequently conducted a region-of-interest (ROI) analysis of the vIFG. This area has been implicated with semantic retrieval processes (Thompson-Schill et al., 1997). To assess the specificity of task-related activity differences between the stimulation conditions, we also assessed activity in two control regions (left dorsal IFG, dIFG; right homolog area of the vIFG) that have been implicated with more general selection or phonological retrieval processes (dIFG; Nagel et al., 2008) and more effortful processing during semantic retrieval (right vIFG; Thompson-Schill et al., 1997). For the ROI analysis $10 \mathrm{~mm}$ spheres were created using the WFU pick-atlas (Maldjian et al., 2003) centered around Talairach coordinates - 48/23/2 (left vIFG; BA 45/47) and $-42 / 30 / 23$ (left dIFG; BA 46/9) according to Nagel et al. (2008). The right hemisphere homolog vIFG ROI was the mirror image of the left vIFG. For both imaging sessions, mean beta activity (from the contrast "word generation > baseline") was extracted from the respective ROIs and compared using pairwise $t$ tests.

Resting-state data analysis. Resting-state fMRI data analysis was performed using LIPSIA software (Lohmann et al., 2001). ECM is a graphbased approach that was chosen because it does not depend on a priori assumptions and for its computational efficiency (see Lohmann et al., 2010a for a detailed description of the method). The ECM approach is fundamentally different compared with other resting-state data analysis approaches such as Independent Component Analysis (ICA; Smith et al., 2009) or seed-based approaches (Biswal et al., 2010). For example, ICA aims at delineating maximally independent temporal or spatial components within fMRI datasets and requires a priori specifications of the number of components and substantial a posteriori selection of valid components (Margulies et al., 2010). In contrast, ECM quantitatively characterizes complex network structures without requiring any assumptions about the underlying network structure, while taking into account the interconnectedness of central brain regions ("hubs") across the entire brain (Bonacich, 2007). Contrary to ICA, ECM does not per se aim to delineate independent cortical networks. Hubs identified by ECM, which are by themselves strongly connected to other central areas across the entire brain, may thus belong to networks supporting different but interacting cognitive processes (Lohmann et al., 2010a). ECM attributes a centrality value to each brain voxel, with higher values indicating voxels that are more strongly connected to other voxels central within a network (Lohmann et al., 2010a; Taubert et al., 2011). In the context of resting-state data analysis, ECM can be used to compare spectral coherences between fMRI time series in different frequency bands (Salvador et al., 2005; Lohmann et al., 2010a). To specifically investigate lowfrequency fluctuations, we focused on spectral ECM (Lohmann et al., 2010a) of frequency bands from 0.03 to $0.06 \mathrm{~Hz}$ (Achard et al., 2006) that have been shown to be particularly valuable for assessing interregional dependencies (Salvador et al., 2005). Preprocessing steps included motion and slice time correction, spatial normalization to the LIPSIA template (voxel resolution $3 \times 3 \times 3 \mathrm{~mm}^{3}$ ), bandpass filtering at $1 / 90 \mathrm{~s}$, and spatial smoothing with a Gaussian kernel of $6 \times 6 \times 6 \mathrm{~mm}$. We restricted our analysis to the gray matter using a binary mask obtained from a study-specific template created with FSL after segmenting the anatomical T1 images (FSL-VBM, http://www.fmrib.ox.ac.uk/fsl; Smith et al., 2004). 
Due to excessive motion in one of their two resting-state scans, two participants were excluded from the analysis. Individual voxelwise spectral coherence analysis was conducted for frequency intervals of $0.03,0.04,0.05$, and $0.06 \mathrm{~Hz}$ for the atDCS and sham conditions separately. After $z$-transformation of the resulting matrices to obtain a Gaussian normal distribution (Lohmann et al., 2010a), the four spectral bands were averaged on an individual basis and the resulting mean images for two stimulation conditions were compared by a whole-brain pairwise $t$ test. Clusters were considered significant at $p<0.05$, corrected for multiple comparisons using a Monte-Carlo simulation (Lohmann et al., 2010a).

In addition, we used LIPSIA to conduct a seed-based functional connectivity analysis of the resting-state data (atDCS condition) to further investigate whether the clusters found in the above ECM analysis during atDCS are part of a network that is connected with the stimulated left IFG. Similar to the task-specific fMRI analysis, we used peak voxels in the left vIFG and dIFG (Nagel et al., 2008) as centers of spheres with a volume of 33 voxels (Lohmann et al., 2010b). The preprocessed fMRI time series was averaged across voxels within the respective spheres to obtain Pearson's correlation coefficients with all other voxels in the gray matter mask. The resulting voxelwise correlation coefficients were normalized using the Fisher $r$-to- $z$ transformation.

Positive and negative mood ratings. Two self-report rating scales were administered immediately before and after the two scanning sessions to assess mood and positive and negative affect [Visual Analog Mood Scales, VAMS (Folstein and Luria, 1973); Positive and Negative Affect Scales, PANAS (Watson et al., 1988)]. The PANAS assesses positive and negative affect (10 items each) on a scale ranging from 1 to 5 , where higher values describe more positive or negative feelings. The VAMS comprises seven items that assess positive (2 items) and negative (5 items) mood using a visual analog scale (range $0-100$ ). Higher values indicate more positive or negative mood ratings.

\section{Results}

All participants tolerated the stimulation well and none reported side effects during or after stimulation. As in previous studies that used $1 \mathrm{~mA}$ (Gandiga et al., 2006), subjects could not differentiate between active and sham stimulation, as indicated by a post-study questionnaire.

\section{Anodal stimulation improved semantic word generation}

In line with previous studies that showed improved word-retrieval following atDCS of the left IFG (Iyer et al., 2005; Cattaneo et al., 2011; Holland et al., 2011), participants produced significantly more correct responses during atDCS compared with sham (mean \pm SD atDCS: $55.7 \pm 2.9$, sham: $53.3 \pm 3.6$; two-tailed paired $t$ test, $\left.t_{(19)}=2.94, p=0.008\right)$. Response times for correct responses did not differ between the two stimulation

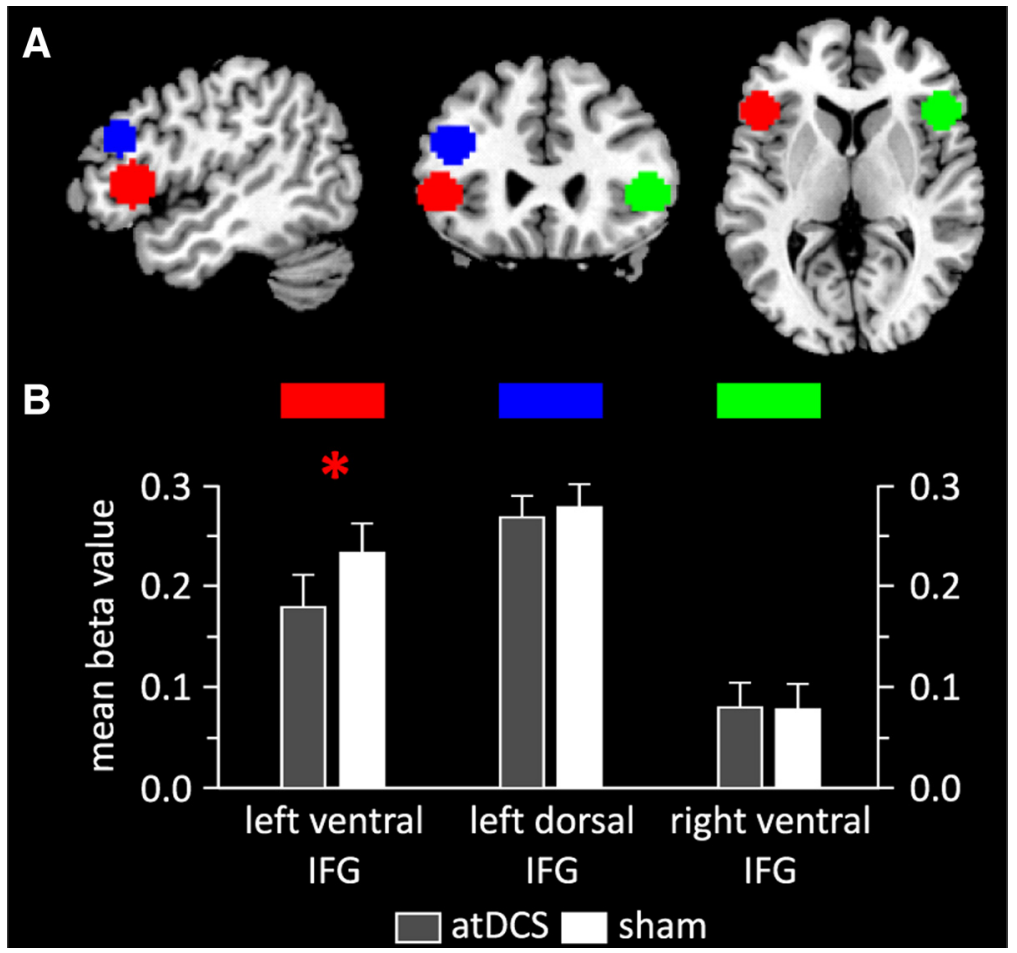

Figure 3. ROI analysis. $\boldsymbol{A}$, Location of the three a priori ROls: $10 \mathrm{~mm}$ spheres in the left ventral (red) and dorsal (blue) IFG were generated according to Nagel et al. (2008). An additional ROI in the right vIFG (green) constitutes the mirror image of the left vIFG. $B$, Activity levels ( $N=20$ participants; mean \pm SEM beta values from the contrast semantic word generation $>$ baseline) in the respective ROls for the two stimulation conditions.

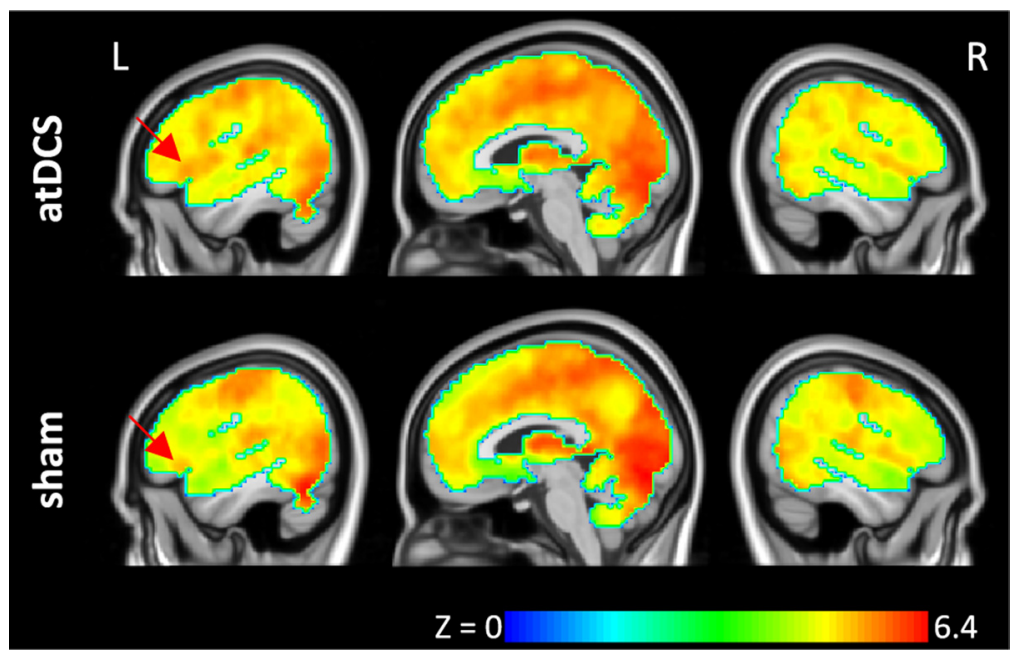

Figure 4. Shows baseline ECM maps for the two stimulation conditions. Under sham stimulation the ventral IFG (red arrow) showed high centrality values (peak $z=0.53$; mean/SEM in the vIFG ROI from the task-related analysis $z=0.48 \pm 0.09$ ). Please note that the maximum centrality value across the entire brain was $z=0.64$. Under atDCS, peak centrality values in the vIFG ROI further increased to $z=0.56$ (mean/SEM $0.50 \pm 0.09$ ). R, Right; L, Left. MNI coordinates of sagittal slices $x=-49 / 3 / 49$. conditions (mean \pm SD atDCS: $851.2 \pm 182.5 \mathrm{~ms}$, sham: $\left.865.5 \pm 267.4 \mathrm{~ms}, t_{(19)}=0.43, p=0.67\right)$.

fMRI revealed specific task-related activity reductions during atDCS versus sham $\mathrm{tDCS}$

As in previous studies that used similar word generation designs (Meinzer et al., 2009, 2012) a whole-brain comparison of correct semantic word generation trials with the baseline condition (saying the word "rest" aloud) elicited a reliable and highly consistent 


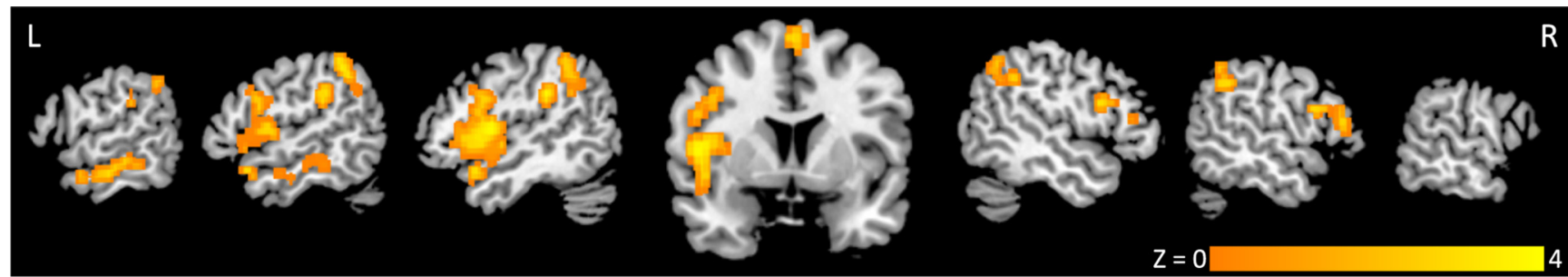

Figure 5. Shows areas that exhibited enhanced connectivity (Eigenvector centrality) during atDCS compared with sham ( $N=18$ participants; two participants had to be excluded due to excessive movement during resting-state fMRI). L, Left; $\mathrm{R}$, right. Clusters were significant at $p<0.05$, corrected for multiple comparisons using Monte-Carlo simulation, MNI coordinates of sagittal slices $z=$ $50 / 55 / 60$ (right hemisphere), $z=-50 /-55 /-60$ (left hemisphere), coronal slice $y=5$.

pattern of task-related activity for both conditions. Most pronounced activity was found in the lateral (ventral and dorsal) IFG and medial frontal areas, but also left posterior temporal and parietal areas and the right inferior frontal gyrus [Fig. 2 illustrates the activity patterns associated with correct word generation trials vs baseline during the two stimulation conditions; $p<0.0005$, corrected for multiple comparisons using the false discovery rate (Genovese et al., 2002)].

To assess the specificity of task-related activity differences, we conducted a ROI analysis that focused on the left vIFG and two control regions (left dIFG; right homolog area of the vIFG), that have been implicated in more general selection or phonological retrieval processes (dIFG; Nagel et al., 2008) and more effortful processing during semantic retrieval (right vIFG; ThompsonSchill et al., 1997). As hypothesized, we found selectively reduced activity in the left vIFG during atDCS versus sham tDCS (paired two-tailed $t$ test $t_{(19)}=2.58, p=0.02$ ), while no differences were found in the control ROIs (left dIFG: $t_{(19)}=0.49, p=0.62$; right vIFG: $t_{(19)}=-0.12, p=0.90$; see Fig. 3 for details and location of ROIs). No correlation was found between differences in beta values and performance differences (sham vs atDCS). We also conducted an exploratory second level whole-brain analysis to assess whether there were activity reductions (sham $>$ atDCS) or increases (atDCS $>$ sham) outside of the stimulated IFG. However, neither of these comparisons yielded differences that survived a corrected threshold.

Anodal stimulation increased language network connectivity ECM analysis of low-frequency fluctuations $(0.03-0.06 \mathrm{~Hz})$ during task-independent resting-state fMRI revealed increased connectivity, as indicated by higher eigenvector centrality values, in the IFG and other major hubs overlapping with the bilateral language network (Price, 2000; Poeppel and Hickok, 2004) during atDCS compared with sham stimulation (see Fig. 4 for baseline ECM maps during atDCS and sham; Fig. 5 and Table 1 detail differences between the stimulation conditions). In particular, the cluster with the strongest tDCS-induced increase was found at the stimulation site, i.e., the left IFG and anterior insula. Additional significant clusters were located in bilateral inferior parietal, dorsolateral and medial prefrontal regions as well as the left middle temporal gyrus. Reduced connectivity was found only in more posterior sensory-motor and occipital regions (Table 1). In addition, we assessed which areas with strongly modulated resting-state activity overlapped with clusters found in the task-specific analysis. Next to the left and right IFG ROIs, activity in one additional cluster in the supplementary motor area (SMA; Table 1) overlapped with the task-related activity. When we compared task-related activity during the two stimulation conditions in this area, no activity
Table 1. Results of the ECM analysis: location and size of clusters showing enhanced (positive z-score) or reduced (negative Z-score) Eigenvector Centrality during atDCS compared with sham (paired $t$ test; $p<0.05$, corrected for multiple comparisons using a Monte-Carlo simulation)

\begin{tabular}{lrrrrrr}
\hline Anatomical location & BA & $k$ & $Z$ & $x$ & $y$ & $z$ \\
\hline Increased connectivity in language-related areas & & & & & & \\
$\quad$ L inferior frontal gyrus/insula & 13 & 376 & 4.46 & -29 & 27 & 15 \\
L inferior parietal gyrus & 40 & 71 & 4.35 & -43 & -48 & 34 \\
L middle frontal gyrus & 8 & 35 & 4.11 & -38 & 33 & 45 \\
L superior frontal gyrus & 8 & 50 & 3.95 & -12 & 42 & 42 \\
L inferior parietal gyrus & 40 & 40 & 3.82 & -49 & -33 & 28 \\
R superior frontal gyrus & 10 & 44 & 3.73 & 20 & 42 & 26 \\
R inferior parietal gyrus & 40 & 31 & 3.60 & 52 & -45 & 37 \\
L/R medial frontal gyrus & 6 & 44 & 3.52 & 0 & 0 & 61 \\
L middle temporal gyrus & 21 & 34 & 3.48 & -58 & -21 & -10 \\
R inferior frontal gyrus & 9 & 35 & 3.42 & 49 & 6 & 23 \\
Reduced connectivity in sensory-motor and visual areas & & & & & & \\
L postcentral gyrus & 5 & 194 & -4.09 & -29 & -45 & 64 \\
L precentral gyrus & 4 & 35 & -3.34 & -23 & -24 & 70 \\
R superior parietal gyrus & 7 & 39 & -3.43 & 17 & -54 & 59 \\
R postcentral gyrus & 3 & 32 & -3.56 & 52 & -18 & 39 \\
& & 38 & -3.20 & 46 & -21 & 53 \\
R posterior cingulate gyrus & 31 & 37 & -3.06 & 9 & -36 & 39 \\
R lingual gyrus & 18 & 121 & -4.95 & 9 & -72 & 6 \\
L lingual gyrus & 17 & 51 & -3.42 & -14 & -87 & 4 \\
R fusiform gyrus & 37 & 54 & -4.73 & 23 & -48 & -16 \\
L fusiform gyrus & 37 & 94 & -3.75 & -46 & -60 & -18 \\
\hline
\end{tabular}

hemi, Hemisphere; $\mathrm{R}$, right; $\mathrm{L}$, left; $k=$ cluster extent (number of voxels); $x / y / z$, coordinates of peak voxel in significant clusters (Talairach space).

differences were found (mean \pm SEM beta values sham: $0.15 \pm 0.03$, atDCS: $0.13 \pm 0.02 ; t_{(19)}=1.0, p=0.31$ ).

The seed-based analysis revealed partially overlapping, but also divergent networks that correlated positively with the respective IFG seeds (see Fig. 6 for details). In particular, the strongest positive correlations of the vIFG ROI were found with bihemispheric perisylvian areas including the vIFG, insula, posterior temporal and inferior parietal cortices. Additional clusters of strong correlations were located in the pre-SMA and SMA as well as in the basal ganglia and the cerebellum bilaterally. Negative correlations could only be substantiated for the posterior cingulate cortex/precuneus. The left dIFG ROI showed strong positive correlations with the right dIFG, bilateral dorsolateral prefrontal and inferior and superior parietal as well as posterior temporal cortices, the pre-SMA and the posterior cingulate cortex. No negative correlations were found.

\section{Self-reported mood}

No differences were found between pre-post ratings for the two stimulation conditions, indicating that mood was not affected by the respective stimulation conditions (VAMS: atDCS positive/ negative score: $t_{(19)}=0.25 / 0.41, p=0.79 / 0.68$; sham positive/ 
negative score: $t_{(19)}=-0.09 /-1.01, p=$ $0.92 / 0.32)$; PANAS: atDCS positive/negative score: $t_{(19)}=-0.06 /-0.84, p=0.95 /$ 0.40 ; sham positive/negative score: $t_{(19)}=$ $0.87 /-1.10, p=0.39 / 0.28)$. The direct comparison of pre-post differences between the stimulation conditions also revealed no differences (VAMS: positive/ negative score $t_{(19)}=0.34 /-0.55, p=0.73 /$ 0.58 ; PANAS: positive/negative score $t_{(19)}=$ $-0.49 / 0.72, p=0.62 / 0.47$; see Table 2 for details).

\section{Discussion}

Despite the potential major impact of tDCS on cognitive neuroscience research and implications for neurorehabilitation, the neural correlates associated with beneficial behavioral effects of atDCS have rarely been studied. Only few studies assessed the effects of tDCS on brain activity in the language (Holland et al., 2011) or motor domain (Antal et al., 2011; Polanía et al., 2011, 2012; Zheng et al., 2011).

However, none of the previous studies addressed higher cognitive functions and, at the same time, provided comprehensive information on tDCS-induced task-related local activity changes and functional connectivity modulations.

In the present study, we assessed neural signatures of improved language function induced by atDCS over a core region of the human language system, the left IFG (Lohmann et al., 2010b), at the local and functional network level. In line with previous behavioral studies (Iyer et al., 2005; Cattaneo et al., 2011) atDCS resulted in highly significant improvement of semantic wordretrieval compared with sham stimulation. As hypothesized, improved word-retrieval during intrascanner atDCS was associated with significantly reduced task-related activity in the ventral portion of the left IFG, an area crucial for controlled semantic retrieval processes (Thompson-Schill et al., 1997). Reduced activity may be related to more efficient processing in task-critical areas as suggested by two recent studies. In particular, Holland et al. (2011) showed that improved picture naming performance after left IFG stimulation was correlated with reduced activity in the IFG and premotor cortex. In our own study, we found no correlation between beta values and performance differences. However, our healthy young participants performed at a relatively high level during both stimulation conditions and even though the difference in correct responses between the two conditions was consistent and highly significant, the absolute difference of correct responses in the respective conditions was relatively small with little variance. This may have prevented a similar correlation as reported by Holland et al. (2011). In a second study, Antal et al. (2011) applied atDCS over the primary motor cortex and subjects performed a self-paced finger tapping task. While no activity differences were found at the stimulation site, reduced activity was found in a tightly connected area (SMA) implicated with selfinitiated motor actions (Nachev et al., 2008). In our own study, no activity differences were found between the stimulation conditions for two predefined control ROIs in the vicinity of the stimulation site (left dIFG) and a remote area (right vIFG), that were both active during the task and no changes were found in the SMA, that was active during task-related fMRI and showed an activity modulation during the resting-state scans under atDCS. Thus, the high
Table 2. Details of mood and affect ratings

\begin{tabular}{llllll}
\hline & \multicolumn{2}{l}{ Before stimulation } & & \multicolumn{2}{l}{ After stimulation } \\
\cline { 2 - 3 } & Sham & Anodal & & Sham & Anodal \\
\hline PANAS & & & & & \\
$\quad$ Positive score & $2.82 \pm 0.13$ & $2.89 \pm 0.11$ & & $2.88 \pm 0.12$ & $2.89 \pm 0.13$ \\
$\quad$ Negative score & $1.18 \pm 0.06$ & $1.15 \pm 0.03$ & & $1.15 \pm 0.06$ & $1.10 \pm 0.03$ \\
VAMS & & & & \\
$\quad$ Positive score & $0.65 \pm 0.05$ & $0.62 \pm 0.05$ & & $0.65 \pm 0.08$ & $0.63 \pm 0.05$ \\
$\quad$ Negative score & $0.10 \pm 0.05$ & $0.09 \pm 0.02$ & & $0.09 \pm 0.09$ & $0.12 \pm 0.07$ \\
\hline Values are mean \pm SEM. & & & &
\end{tabular}

regional specificity of our results is in line with specific neural priming effects in left frontal areas in the study by Holland et al. (2011).

Indeed, the finding that "less is more" has been described in a number of previous studies: Reduced activity in circumscribed task-related areas or more focal processing has been implicated with consolidation of motor learning (Pascual-Leone et al., 1994) and superior memory performance (Gonsalves et al., 2005). In the language domain, increasing proficiency in a newly learned second language is associated with more focal left-lateralized activity in prefrontal cortices, resembling the network active during language processing in the mother tongue (Abutalebi, 2008). Furthermore, increased bilateral IFG activity is associated with reduced semantic word-generation performance in healthy aging (Meinzer et al., 2009, 2012), and even in young adults when task demands are increased (Thompson-Schill et al., 1997). Other studies found increased activity after tDCS. For example, Lindenberg et al. (2010) assessed brain activity patterns during a motor task in patients with post-stroke hemiparesis before and after five consecutive days of physical and occupational therapy, either with or without simultaneous (bihemispheric) tDCS. Training in combination with tDCS was associated with activity increases in the ipsilesional motor cortex. However, the results of this study cannot be easily compared with our own results because of a number of study-specific factors. Most importantly, this study assessed long-term effects of a multisession intervention (tDCS + motor training) in a group of chronic stroke patients. In contrast, we investigated short-term changes in functional activity induced by tDCS in healthy subjects. Note that our own results 
are in line with the study by Holland et al. (2011) who used a similar design and also included healthy subjects only.

The analysis of task-related activity changes was complemented by a whole-brain functional connectivity analysis of resting-state fMRI. We focused on low-frequency oscillations which are thought to reflect underlying anatomical connectivity and may provide a physiological substrate for information processing at the network level (Achard and De Schutter, 2006). Major hubs of the slow-frequency network are critical for cognition and recently, the left IFG has been described as one of the key nodes of a basic network for language processing (Lohmann et al., 2010b). We used a graph-based ECM analysis that has previously been applied to resting-state fMRI and successfully differentiated physiological state-dependent functional network differences (hunger vs satiety; Lohmann et al., 2010a) and connectivity changes after motor learning (Taubert et al., 2011). In the present study, we show that the vIFG was already a central hub in the sham condition (Fig. 4) and its centrality was further enhanced under atDCS as indicated by significantly higher eigenvector centrality values compared with sham stimulation (Fig. 5). However, stimulation effects were not limited to the stimulation site, but extended to a number of different areas as demonstrated by the ECM analysis. These areas were functionally connected with the IFG as confirmed by an additional seed-based functional connectivity analysis. Interestingly, areas with enhanced eigenvector centrality under atDCS were functionally connected with the ventral and dorsal IFG (Fig. 6) and the combined networks almost completely overlapped with areas that showed enhanced eigenvector centrality under atDCS. This is most likely explained by the fact that even though the center of the tDCS electrode was positioned over the left vIFG, a major portion of the dIFG was also stimulated due to the relatively large size of the electrode which covered both the vIFG and dIFG.

Based on the present study we cannot make assumptions about the exact functional role of areas that showed increased centrality in the ECM analysis. However, we would like to point out that these areas overlap with brain regions that have previously been associated with critical aspects of language processing (Price, 2000; Poeppel and Hickok, 2004; Catani et al., 2005). In addition to classical language areas implicated with semantic, phonological, and syntactic processing (bilateral IFG and inferior parietal lobe, left middle temporal gyrus), this network comprised nodes associated with working memory or attentional processes (dorsolateral prefrontal cortex, medial frontal cortex; Price, 2000; Poeppel and Hickok, 2004). Similar results with regard to network effects have been reported after stimulation of the primary motor cortex (Polanía et al., 2011). In this previous study, atDCS compared with sham increased resting-state connectivity assessed by electroencephalography in a larger motor-related network including primary motor, premotor, and sensory-motor cortices.

In conclusion, the combination of intrascanner stimulation with two complementing types of task-specific and taskindependent analyses allowed unprecedented insights into local and system level effects of atDCS. It has been suggested previously that local effects at the stimulation site may be mediated by modulation of resting membrane potentials, thus, reducing the threshold necessary for depolarization of the underlying taskrelevant neural populations (Stagg and Nitsche, 2011). This in turn may facilitate the responsiveness of a subset of highly specific task-relevant neurons, as in our own study, the left vIFG, even though larger cortical areas are stimulated. In addition, the stimulation alters connectivity within a larger set of anatomically and functionally connected brain regions. These networks seem to depend on the primary stimulation site; i.e., stimulation of primary motor areas results in specific upregulation of a motorrelated network (Polanía et al., 2011), while stimulation of the language system enhances connectivity of a different subset of language-associated brain regions. Moreover, even though previous modeling studies have shown that atDCS results in modulation of large cortical areas (e.g., Wagner et al., 2007), this does not result in an unspecific upregulation of task-related activity patterns. Rather, our results suggest that during task performance, enhanced connectivity in a given network provides the basis for enhanced neural efficiency in highly specific brain areas critical for task performance. These areas may be located at the stimulation site (as in our own study) or even in distant but tightly connected regions (Antal et al., 2011). This observation may also have major implications for treating neurological conditions caused by different types of lesions. In the future, targeting major hubs of spared network components in combination with symptom-oriented treatment may help to facilitate recovery of function.

\section{References}

Abutalebi J (2008) Neural aspects of second language representation and language control. Acta Psychol (Amst) 128:466-478.

Achard P, De Schutter E (2006) Complex parameter landscape for a complex neuron model. PLoS Comput Biol 2:e94.

Achard S, Salvador R, Whitcher B, Suckling J, Bullmore E (2006) A resilient, low-frequency, small-world human brain functional network with highly connected association cortical hubs. J Neurosci 26:63-72.

Antal A, Nitsche MA, Kruse W, Kincses TZ, Hoffmann KP, Paulus W (2004) Direct current stimulation over V5 enhances visuomotor coordination by improving motion perception in humans. J Cogn Neurosci 16:521-527.

Antal A, Polania R, Schmidt-Samoa C, Dechent P, Paulus W (2011) Transcranial direct current stimulation over the primary motor cortex during fMRI. Neuroimage 55:590-596.

Biswal BB, Mennes M, Zuo XN, Gohel S, Kelly C, Smith SM, Beckmann CF, Adelstein JS, Buckner RL, Colcombe S, Dogonowski AM, Ernst M, Fair D, Hampson M, Hoptman MJ, Hyde JS, Kiviniemi VJ, Kötter R, Li SJ, Lin CP, et al. (2010) Toward discovery science of human brain function. Proc Natl Acad Sci U S A 107:4734-4739.

Bonacich P (2007) Some unique properties of eigenvector centrality. Social Networks 29:555-564.

Catani M, Jones DK, ffytche DH (2005) Perisylvian language networks of the human brain. Ann Neurol 57:8-16.

Cattaneo Z, Pisoni A, Papagno C (2011) Transcranial direct current stimulation over Broca's region improves phonemic and semantic fluency in healthy individuals. Neuroscience 183:64-70.

Flöel A, Rösser N, Michka O, Knecht S, Breitenstein C (2008) Noninvasive brain stimulation improves language learning. J Cogn Neurosci 20:1415-1422.

Flöel A, Meinzer M, Kirstein R, Nijhof S, Deppe M, Knecht S, Breitenstein C (2011) Short-term anomia training and electrical brain stimulation. Stroke 42:2065-2067.

Flöel A, Suttorp W, Kohl O, Kurten J, Lohmann H, Breitenstein C, Knecht S (2012) Non-invasive brain stimulation improves object-location learning in the elderly. Neurobiol Aging. Advance online publication. Retrieved September 2011. doi.org/10.1016/j.neurobiolaging.2011.05.007.

Folstein MF, Luria R (1973) Reliability, validity and clinical application of the visual analogue mood scales. Psychol Med 3:479-486.

Gaab N, Gabrieli JD, Glover GH (2007) Assessing the influence of scanner background noise on auditory processing. I. An fMRI study comparing three experimental designs with varying degrees of scanner noise. Hum Brain Mapp 28:703-720.

Gandiga PC, Hummel FC, Cohen LG (2006) Transcranial DC stimulation (tDCS): a tool for double-blind sham-controlled clinical studies in brain stimulation. Clin Neurophysiol 117:845-850.

Genovese CR, Lazar NA, Nichols T (2002) Thresholding of statistical maps in functional neuroimaging using the false discovery rate. Neuroimage 15:870-878. 
Gonsalves BD, Kahn I, Curran T, Norman KA, Wagner AD (2005) Memory strength and repetition suppression: multimodal imaging of medial temporal cortical contributions to recognition. Neuron 47:751-761.

Holland R, Leff AP, Josephs O, Galea JM, Desikan M, Price CJ, Rothwell JC, Crinion J (2011) Speech facilitation by left inferior frontal cortex stimulation. Curr Biol 21:1403-1407.

Iyer MB, Mattu U, Grafman J, Lomarev M, Sato S, Wassermann EM (2005) Safety and cognitive effect of frontal DC brain polarization in healthy individuals. Neurology 64:872-875.

Lindenberg R, Renga V, Zhu LL, Nair D, Schlaug G (2010) Bihemispheric brain stimulation facilitates motor recovery in chronic stroke patients. Neurology 75:2176-2184.

Lohmann G, Müller K, Bosch V, Mentzel H, Hessler S, Chen L, Zysset S, von Cramon DY (2001) LIPSIA - a new software system for the evaluation of functional magnetic resonance images of the human brain. Comput Med Imaging Graph 25:449-457.

Lohmann G, Margulies DS, Horstmann A, Pleger B, Lepsien J, Goldhahn D, Schloegl H, Stumvoll M, Villringer A, Turner R (2010a) Eigenvector centrality mapping for analyzing connectivity patterns in FMRI data of the human brain. PLoS One 5:e10232.

Lohmann G, Hoehl S, Brauer J, Danielmeier C, Bornkessel-Schlesewsky I, Bahlmann J, Turner R, Friederici A (2010b) Setting the frame: the human brain activates a basic low-frequency network for language processing. Cereb Cortex 20:1286-1292.

Maldjian JA, Laurienti PJ, Kraft RA, Burdette JH (2003) An automated method for neuroanatomic and cytoarchitectonic atlas-based interrogation of fMRI data sets. Neuroimage 19:1233-1239.

Mannhaupt HR (1983) Produktionsnormen für verbale Reaktionen zu 40 geläufigen Kategorien. Sprache und Kognition 4:264-278.

Margulies DS, Böttger J, Long X, Lv Y, Kelly C, Schäfer A, Goldhahn D, Abbushi A, Milham MP, Lohmann G, Villringer A (2010) Resting developments: a review of fMRI post-processing methodologies for spontaneous brain activity. MAGMA 23:289-307.

Meinzer M, Flaisch T, Wilser L, Eulitz C, Rockstroh B, Conway T, GonzalezRothi L, Crosson B (2009) Neural signatures of semantic and phonemic fluency in young and old adults. J Cogn Neurosci 21:2007-2018.

Meinzer M, Seeds L, Flaisch T, Harnish S, Cohen ML, McGregor K, Conway T, Benjamin M, Crosson B (2012) Impact of changed positive and negative task-related brain activity on word-retrieval in aging. Neurobiol Aging. Advance online publication. Retrieved September 2011. doi:10.1016/j.neurobiolaging.2010.06.020.

Nachev P, Kennard C, Husain M (2008) Functional role of the supplementary and pre-supplementary motor areas. Nat Rev Neurosci 9:856-869.

Nagel IE, Schumacher EH, Goebel R, D’Esposito M (2008) Functional MRI investigation of verbal selection mechanisms in lateral prefrontal cortex. Neuroimage 43:801-807.

Pascual-Leone A, Grafman J, Hallett M (1994) Modulation of cortical mo- tor output maps during development of implicit and explicit knowledge. Science 263:1287-1289.

Poeppel D, Hickok G (2004) Towards a new functional anatomy of language. Cognition 92:1-12.

Polanía R, Nitsche MA, Paulus W (2011) Modulating functional connectivity patterns and topological functional organization of the human brain with transcranial direct current stimulation. Hum Brain Mapp 32:1236-1249.

Polania R, Paulus W, Nitsche MA (2012) Modulating cortico-striatal and thalamo-cortical functional connectivity with transcranial direct current stimulation. Hum Brain Mapp. Advance online publication. Retrieved November 2011. doi:10.1002/hbm.21380.

Price CJ (2000) The anatomy of language: contributions from functional neuroimaging. J Anat 197:335-359.

Reis J, Schambra HM, Cohen LG, Buch ER, Fritsch B, Zarahn E, Celnik PA, Krakauer JW (2009) Noninvasive cortical stimulation enhances motor skill acquisition over multiple days through an effect on consolidation. Proc Natl Acad Sci U S A 106:1590-1595.

Salvador R, Suckling J, Schwarzbauer C, Bullmore E (2005) Undirected graphs of frequency-dependent functional connectivity in whole brain networks. Philos Trans R Soc Lond B Biol Sci 360:937-946.

Smith SM, Jenkinson M, Woolrich MW, Beckmann CF, Behrens TE, Johansen-Berg H, Bannister PR, De Luca M, Drobnjak I, Flitney DE, Niazy RK, Saunders J, Vickers J, Zhang Y, De Stefano N, Brady JM, Matthews PM (2004) Advances in functional and structural MR image analysis and implementation as FSL. Neuroimage 23 [Suppl 1]:S208-S219.

Smith SM, Fox PT, Miller KL, Glahn DC, Fox PM, Mackay CE, Filippini N, Watkins KE, Toro R, Laird AR, Beckmann CF (2009) Correspondence of the brain's functional architecture during activation and rest. Proc Natl Acad Sci U S A 106:13040-13045.

Stagg CJ, Nitsche MA (2011) Physiological basis of transcranial direct current stimulation. Neuroscientist 17:37-53.

Taubert M, Lohmann G, Margulies DS, Villringer A, Ragert P (2011) Longterm effects of motor training on resting-state networks and underlying brain structure. Neuroimage 57:1492-1498.

Thompson-Schill SL, D’Esposito M, Aguirre GK, Farah MJ (1997) Role of left inferior prefrontal cortex in retrieval of semantic knowledge: a reevaluation. Proc Natl Acad Sci U S A 94:14792-14797.

Wagner T, Valero-Cabre A, Pascual-Leone A (2007) Noninvasive human brain stimulation. Annu Rev Biomed Eng 9:527-565.

Watson DC, Clark MA, Tellegen A (1988) Development and validation of brief measures of positive and negative affect: The PANAS scales. J Pers Soc Psychol 54:1063-1070.

Zheng X, Alsop DC, Schlaug G (2011) Effects of transcranial direct current stimulation (tDCS) on human regional cerebral blood flow. Neuroimage $58: 26-33$. 